Original Article

\title{
Comparison of Inflammatory Markers like C - Reactive Protein, Total Leukocyte Count and Erythrocyte Sedimentation Rate in Type II Diabetes M ellitus
}

\author{
Aditya M ungamuri ${ }^{1}$, Sunil KumarY ${ }^{2}$, Suchetha Kumari N. ${ }^{3}$, Ullal Harshini Devi ${ }^{4}$ \\ ${ }^{1,}$ U G Student, ${ }^{2}$ Additional Professor, Dept. of Pathology, ${ }^{3}$ Professor, Dept. of Biochemistry, ${ }^{4}$ Central Research Laboratory, \\ KSHegde M edical Academy, M angalore - 575018. \\ Corresponding Author: Sunil KumarY., Additional Professor, Dept. of Pathology, K. S. Hegde Medical Academy, Deralakatte, Mangalore \\ - 575018. E-mail : drsunilkumary@rediffmail.com
}

Received

: 22.07.2017

Review Completed : 29.11.2017

Accepted

:02.02.2018

Keywords : Diabetes mellitus, Inflammatory markers, C-Reactive Protein, Total Leucocyte Count, Erythrocyte Sedimentation Rate.

\begin{tabular}{|c|}
\hline Access this article online \\
\hline Quick Response Code \\
\hline
\end{tabular}

\begin{abstract}
:
Background: Diabetes M ellitus (DM) has become an epidemic in the 21st century where India leads the world with largest number of patients. There is increasing evidence that inflammation is closely involved in the pathogenesis of type 2 diabetes and associated complications such as dyslipidaemia and atherosclerosis. M any previous studies indicate inflammatory markers like CRP, IL-6, IL-8, TNF-alpha, fibrinogen, total sialic acid, ceruloplasmin and total leucocyte count (TLC) are raised in Type II Diabetes M ellitus. However, not many studies have done association of ESR and TLC in DM.
\end{abstract}

Aims: In the present study inflammatory markers like CRP, total leukocyte count and ESR were compared in diabetic and non-diabetic patients.

M aterials and M ethods: $5 \mathrm{~mL}$ of venous blood was taken from the study subjects.CRP, TLC and ESR was estimated.

Results: There was a significant rise in the CRP, TLC and ESR values seen in patients diagnosed with type II diabetes mellitus when compared to normal individuals. All three parameters (CRP, TLC, and ESR) were raised in a total of 7 cases (14\%). 14 cases (28\%) showed elevated levels of both CRP and ESR. A significant 8 cases (16\%) showed elevation of CRP alone. There was a rise of ESR alone in 5 cases (10\%).

Conclusion: CRP, TLC and ESR are elevated in diabetic patients in comparison to normal individuals. These increase the risk of diabetic related complications like atherosclerosis and dyslipaedemia. Hence, anti-inflammatory drugs in combination with antidiabetic treatment can delay these complications.

\section{Introduction}

Diabetes Mellitus is a metabolic disorder characterised by chronic hyperglycaemia with disturbances of carbohydrate, fat and protein metabolism resulting from defects in insulin secretion, insulin action or both. ${ }^{1}$ Type II Diabetes Mellitus (DM2) is the most common form of diabetes. The 'top' three countries in terms of the number of DM 2 individuals with diabetes are India (31.7 million in 2000; 79.4 million in 2030), China (20.8 million in 2000; 42.3 million in 2030); and USA (17.7 million in 2000; 30.3 million in 2030). ${ }^{2}$ Clearly, DM 2 has become an epidemic in the 21st century where India leads the world with largest number of diabetic subjects. The International Diabetes
Federation (IDF) estimates the total number of diabetic subjects to be around 40.9 million in India and this is further set to rise to 69.9 million by the year $2025 .{ }^{3}$ DM 2 results from the interaction between a genetic predisposition, behavioural and environmental risk factors. ${ }^{4}$ The two metabolic defects that characterize DM 2 are, ${ }^{1}$ decreased ability of peripheral tissues to respond to insulin (insulin resistance) and ${ }^{2}$ beta cell dysfunction that is manifested as inadequate insulin secretion in the face of insulin resistance and hyperglycaemia. ${ }^{5}$ This form of diabetes is most often associated with older age, obesity, family history, previous history of gestational diabetes, physical inactivity and certain ethnicities. About $80 \%$ of 
people with DM 2 are overweight. ${ }^{6}$ Diabetes is associated with long-term complications that affect almost every organ of the body. The disease often leads to blindness, heart, blood vessel disease, stroke, kidney failures, amputations and nerve damage. Uncontrolled diabetes can complicate pregnancy and birth defects are more common in babies born to women with diabetes. ${ }^{6,7,8}$ However, there has been a significant increase in cases of DM 2 under the age of 30 years. This is a great concern as future generations may be burdened with morbidity and mortality at the cost of their productivity, potentially affecting the workforce and healthcare resources of the countriesacross the world. ${ }^{6,9}$, and 10

Wang et al., in 2012 conducted a complete meta-analysis on the role of inflammatory markers in DM2. They concluded that C-Reactive Protein (CRP) and Interleukin-6 had significant association with Diabetes. ${ }^{7}$ However, not many studies have been carried out in world literature to ascertain the role of Total Leucocyte Count (TLC) and Erythrocyte Sedimentation Rate (ESR) in DM2. In the present proposed study our primary attempt is to make a comparative study of the effect of DM 2 on various serum inflammatory markers like $\mathrm{C}$ - reactive protein (CRP), Total Leucocyte Count (TLC) and Erythrocyte Sedimentation Rate (ESR). The results of this study can be of great prognostic value.

\section{M aterials and methods}

The study was approved by the Institutional ethics committee (INST.EC/EC/51/2016-17). This prospective study was conducted in a tertiary hospital of coastal Karnataka. Total of 50 Diabetes and 50 Healthy control subjects were included in the study. The criteria for Control Group include non-Diabetic individuals from general population more than 20 years and who are willing to give the written consent. The criteria for diabetic group include patients diagnosed with DM2 based on international protocol with age group more than 20 years and who are willing to give the written consent. We excluded smokers, alcoholics, hypertensives, obese individuls, previous organ transplants, patients with infections / tissue injury/ recent surgeries/ autoimmune disorders/ ischemic heart disease or malignancy.

Participants were carefully selected based on set inclusion and exclusion criteria. Written Informed Consent was also taken from the subject having explained all details regarding the study the in language understood to them.

$5 \mathrm{~mL}$ of venous blood were taken from the participants and collected in two different vacutainers i.e. Plain and EDTAtreated vacutainers. The plain blood sample was centrifuged at 2500rpm for 10 minutes; serum was separated and used for estimation of CRP (quantitative turbidometric method). Blood collected in the EDTAtreated vacutainer was used to determine Total Leukocyte Count (TLC) and Erythrocyte Sedimentation Rate. TLC was estimated using autoanalyser (BC-5360), whereas ESR was calculated manually using westergren's pipettes.

\section{Statistical Analysis}

The collected information was summarised by using the descriptive statistics such as frequency, percentage, minimum, maximum, mean, standard deviation, median and IQR (Inter-Quartile Range). To compare the TLC (c/cu.mm)(Table 1), ESR (mm/hr) (Table 2), CRP ( $\mathrm{mg} / \mathrm{l}$ )(Table 3) between case and control M ann-Whitney $\mathrm{U}$ test (Inferential statistics) was used. The $p$ value $\varangle$.05 was considered significant.

\section{Results}

A total no. of 100 individuals ( 50 cases and 50 controls) was systemically identified using the prescribed inclusion and exclusion criteria. There were 70 males and 30 females; varying from age groups from 20 to 80 years. M ean age was 45 years.

The following observations were made among the 50 cases. There was a significant rise in the CRP, TLC and ESR values seen in patients diagnosed with type II diabetes mellitus when compared to normal individuals. All three parameters (CRP, TLC, and ESR) were raised in a total of 7 cases (14\%). 14 cases (28\%) showed elevated levels of both CRP and ESR. 1 case (2\%) showed elevated CRP and TLC levels. There were no cases showing increase in TLC with 
ESR. A significant 8 cases (16\%) showed elevation of CRP alone. There was a rise of ESR alone in 5 cases (10\%). No cases were seen with increased TLC alone. Only 15 participants (30\%) showed normal CRP, ESR and TLC values.

The diagramatic representation of the results are shown below in Fig.1

Table 1: Descriptive Statistics and Mann Whitney U test for Total Leucocyte count between case and control subjects. $P<0.05$ is considered statistically significant.

\begin{tabular}{|c|c|c|c|c|c|c|c|c|}
\hline & M aximum & Minimum & Mean & Standard Deviation & Median & IQR & Mann-Whitney U & $P$ value \\
\hline Case & 16100 & 4800 & 8586 & 2557.822 & 8350 & $6950-9500$ & $659.5(Z=4.072)$ & $\varangle .001$ \\
\hline Control & 11400 & 3800 & 6690 & 1568.471 & 6800 & $5450-7425$ & & \\
\hline
\end{tabular}

Table 2 : Descriptive Statistics and M ann Whitney U test for Erythrocyte Sedimentation Rate between case and control subjects. P $\varangle .05$ is considered statistically significant.

\begin{tabular}{|l|c|c|c|c|c|c|c|c|}
\hline & Maximum & Minimum & M ean & Standard Deviation & M edian & IQR & Mann-Whitney U & P value \\
\hline Case & 116 & 3 & 31.98 & 34.731 & 17.50 & $5-50$ & $585(Z=4.628)$ & \multirow{4}{*}{$\varangle .001$} \\
\cline { 1 - 7 } Control & 30 & 3 & 7.18 & 6.110 & 5 & $3-8.5$ & & \\
\hline
\end{tabular}

Table 3: Descriptive Statistics and M ann Whitney U test for $C$ - reactive protein between case and control subjects. $P \varangle 0.05$ is considered statistically significant.

\begin{tabular}{|l|c|c|c|c|c|c|c|c|}
\hline & Maximum & Minimum & Mean & Standard Deviation & Median & IQR & Mann-Whitney U & P value \\
\hline Case & 292.6 & 0.5 & 42.920 & 70.0211 & 8.680 & $1.768-54.412$ & $461(Z=5.443)$ & $<0.001$ \\
\hline Control & 10.2 & 0.5 & 1.877 & 2.0411 & 1.360 & $0.688-1.978$ & & \\
\hline
\end{tabular}

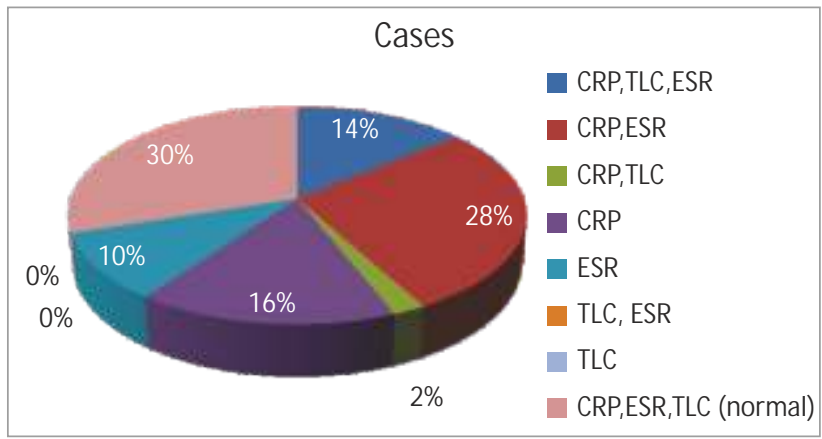

Fig 1 : Pie-chart representing increase in inflammatory markers in cases

\section{Discussion}

Type II Diabetes M ellitus is a global health problem in the $21^{\text {st }}$ century. Inflammation is a key regulator in the pathogenesis of complications associated with Diabetes. Hence, assessment of inflammatory markers might help to follow up all diabetic patients and assess the severity of their complications. Many previous studies indicate inflammatory markers like CRP, IL-6, IL-8, TNF-a, fibrinogen, total sialic acid, ceruloplasmin and TLC are raised in Type II Diabetes Mellitus. ${ }^{7,}{ }^{8}$ However, and not many studies have been done on T2DM with the association of Erythrocyte Sedimentation Rate and Total Leucocyte Count.

In our present study, we have compared the levels of C - reactive protein, Total Leucocyte Count and Erythrocyte Sedimentation Rate with Type II Diabetes M ellitus.

Mugabo $Y$ et al. in the year 2010 demonstrated the role of CRP in the development of diabetic vasculopathy. ${ }^{11}$ Ridker $P$ in the year 2003 concluded that CRP is also an independent indicator of future cardiovascular events that adds prognostic information to lipid screening, metabolic syndrome and Framingham Risk Score. ${ }^{12}$ Our studies showed high levels of CRP in $60 \%$ of the Diabetic patients. The high levels of CRP demonstrated in our study can indicate future cardiovascular diseases in these individuals.

Elias AN and Domurat E in the year 1989 had conducted a study to demonstrate the relationship between ESR and diabetes. ${ }^{13}$ they reported an increase in ESR levels among diabetics. Our present study also shows an increase in ESR levels in $50 \%$ of the test subjects. ESR can be used as an important marker for diagnosis of T2DM. ESR $\geq 100$ $\mathrm{mm} / \mathrm{hr}$ can indicate hidden malignancies which were not diagnosed previously. In our study, 6 cases showed ESR more than 100 . However, these patients were not suffering from any malignancies.

Fu-M ei Chung et al. in the year 2005 demonstrated the role of leukocytes in the development and progression of 
diabetic neuropathy. ${ }^{14}$ Raised TLC can help in diagnosis of any hidden infections in such diabetic patients. In our study, 16\% cases showed increase in TLC levels. These patients were treated with empirical antibiotics. Unfortunately, the long term follow up of these patients could not be done.

\section{Conclusion}

After comparing all three inflammatory markers; $C$ reactive protein, Total leukocyte count and Erythrocyte sedimentation rate in patients of Type II Diabetes M ellitus, we found that all the three parameters are increased in

\section{References}

1. World Health Organisation. Definition, Diagnosis and Classification of Diabetes M ellitus and its complications. Report of a WHO Consultation. Geneva: World Health Organisation, 1999.

2. Wild S, Roglic G, Green A, Sicree R, King H. Global Prevalence of Diabetes: Estimates for the year 2000 and projections for 2030. Diabetes care 2004;27:1047-53

3. Sicree R, Shaw J, Zimmet P. Diabetes and impaired glucose tolerance. In: Gan D, editor. Diabetes Atlas. International Diabetes Federation. $3^{\text {rd }}$ ed. Belgium: International Diabetes Federation; 2006 p. 15-103.

4. NEELJV. Diabetes mellitus: a "thrifty" genotype rendered detrimental by "progress"? AmJ Hum Genet 1962;14:353-62.

5. Vinay Kumar, Abdul K Abbas, Jon C Aster. Robins and Cotran Pathological Basis of Disease. $9^{\text {th }}$ edition: Elsevier Inc; 2015.

6. Singh, Sunitha. The genetics of Type II Diabetes mellitus: a review. J Sci res2011;35-4

7. Wang X, Bao W, Liu J, OuYang Y, Wang D, Rong S et al. inflammatory Markers and Risk Type II Diabetes: A systemic review and metaanalysis. Diabetes care 2012;36:166-75

8. Pradhan A. C-Reactive Protein, IL-6, and risk of developimg Type II these patients in comparison to normal individuals. Among the three, CRP and ESR are significantly elevated inT2DM . These may increase the risk of diabetic related complications like atherosclerosis and dyslipaedemia. Hence, anti-inflammatory drugs in combination with antidiabetic treatment can delay these complications.

\section{Acknowledgement}

I would like to thank Nitte University for funding this project. I would also like to acknowledge the statisticians and the Central Research Laboratory, KSHEM A for carrying out this project.

Diabetes M ellitus. JAM A 2001;286:327.

9. Hu FB, M eigs JB, Li TY, Rifai N, M anson JE. Inflammatory markers and risk of developing Type II Diabetes in women. Diabetes 2004;53:693700.

10. Twig G, Afek A, Shamiss A, Deranze E, Tzur D, Gordon B et al. White Blood Cells Count And Incidence of Type 2 Diabetes in young men. Diabetes care. 2012;36:276-282.

11. M ugabo Y, Li L, Reiner G. The Connection Between C-Reactive Protein and Diabetic vasculopathy. Focus on preclinical findings. Current diabetes reviews. 2010;6(1):27-34.

12. Ridker P. Clinical Application of CRP for Cardiovascular Disease detection and prevention. Circulation. 2003;107(3):363-369.

13. AN Elias, E Domurat. Erythrocyte sedimentation rate in diabetic patients: relationship to glycosylated haemoglobin and serum proteins. J M ed. 1989;20(3-4):297-302.

14. Chung F, Tsai J, Chang D, Shin S, Lee Y. Peripheral Total and Differential Leukocyte Count in Diabetic Neuropathy: The relationship of plasma leptin to leucocytosis. Diabetes care.. 2005;28(7):1710-1717. 